\title{
No Autopsies on COVID-19 Deaths: A Missed Opportunity and the Lockdown of Science
}

\author{
Monica Salerno ${ }^{1,+}{ }^{\dagger}$, Francesco Sessa ${ }^{2,+}+\underset{0}{ }$, Amalia Piscopo ${ }^{3}$, Angelo Montana ${ }^{1}$, Marco Torrisi ${ }^{1}$, \\ Federico Patanè ${ }^{1}$, Paolo Murabito ${ }^{4}$, Giovanni Li Volti ${ }^{5, *(1)}$ and Cristoforo Pomara ${ }^{1, *}$ \\ 1 Department of Medical, Surgical and Advanced Technologies “G.F. Ingrassia”, University of Catania, \\ 95121 Catania, Italy; monica.salerno@unict.it (M.S.); angelomontana49@gmail.com (A.M.); \\ marco.torrisi.1992@gmail.com (M.T.); federicopatane90@gmail.com (F.P.) \\ 2 Department of Clinical and Experimental Medicine, University of Foggia, 71122 Foggia, Italy; \\ francesco.sessa@unifg.it \\ 3 Department of Law, Forensic Medicine, Magna Graecia University of Catanzaro, 88100 Catanzaro, Italy; \\ amaliapiscopo@gmail.com \\ 4 Department of General surgery and medical-surgical specialties, University of Catania, 95121 Catania, Italy; \\ paolo.murabito@unict.it \\ 5 Department of Biomedical and Biotechnological Sciences, University of Catania, 95121 Catania, Italy \\ * Correspondence: livolti@unict.it (G.L.V.); cristoforo.pomara@unict.it (C.P.); \\ Tel.: +39-095-478-1357 or +39-339-304-6369 (G.L.V.); +39-095-378-2153 or +39-333-246-6148 (C.P.) \\ + These authors contributed equally to this work.
}

Received: 12 March 2020; Accepted: 13 May 2020; Published: 14 May 2020

Abstract: Background: The current outbreak of COVID-19 infection, which started in Wuhan, Hubei province, China, in December 2019, is an ongoing challenge and a significant threat to public health requiring surveillance, prompt diagnosis, and research efforts to understand a new, emergent, and unknown pathogen and to develop effective therapies. Despite the increasing number of published studies on COVID-19, in all the examined studies the lack of a well-defined pathophysiology of death among patients who died following COVID-19 infection is evident. Autopsy should be considered mandatory to define the exact cause of death, thus providing useful clinical and epidemiologic information as well as pathophysiological insights to further provide therapeutic tools. Methods: A literature review was performed on PubMed database, using the key terms: "COVID-19", "nCov 19", and "Sars Cov 2". 9709 articles were retrieved; by excluding all duplicated articles, additional criteria were then applied: articles or abstracts in English and articles containing one of the following words: "death", "died", "comorbidity", "cause of death", "biopsy", "autopsy", or "pathological". Results: A total of 50 articles met the inclusion criteria. However, only 7 of these studies reported autopsy-based data. Discussion: The analysis of the main data from the selected studies concerns the complete analysis of 12,954 patients, of whom 2269 died (with a mortality rate of 17.52\%). Laboratory confirmation of COVID-19 infection was obtained in all cases and comorbidities were fully reported in 46 studies. The most common comorbidities were: cardiovascular diseases (hypertension and coronary artery disease), metabolic disorders (diabetes, overweight, or obesity), respiratory disorders (chronic obstructive pulmonary disease), and cancer. The most common reported complications were: acute respiratory distress syndrome (ARDS), acute kidney injury, cardiac injury, liver insufficiency, and septic shock. Only 7 papers reported histological investigations. Nevertheless, only two complete autopsies are described and the cause of death was listed as COVID-19 in only one of them. The lack of postmortem investigation did not allow a definition of the exact cause of death to determine the pathways of this infection. Based on the few histopathological findings reported in the analyzed studies, it seems to be a clear alteration of the coagulation system: frequently prothrombotic activity with consequent thromboembolism was described in COVID-19 patients. As a scientific community, we are called on to face this global threat, and to defeat it with all the available tools necessary. Despite the improvement and reinforcement of any method of study in every field of medicine and science, 
encouraging the autopsy practice as a tool of investigation could also therefore, help physicians to define an effective treatment to reduce mortality.

Keywords: COVID-19; infectious diseases; autopsy; diagnosis

\section{Background}

The current outbreak of COVID-19 infection, which started in Wuhan, Hubei province, China, in December 2019 is an ongoing challenge and a significant threat to public health requiring surveillance, prompt diagnosis, research efforts to understand a new, emergent, and unknown pathogen and to develop effective interventions [1-3].

Despite the increasing number of published studies on COVID-19, there is an evident lack, in all the examined studies, of a well-defined pathophysiology of death among patients who died following COVID-19 infection.

The loss of potential key information about the real mechanisms underlying death due to COVID-19 infections does not allow a real evaluation of COVID-19 mortality, which could even be overestimated given that the precise cause of death remains elusive.

The autopsy should be considered mandatory in order to define the exact cause of death, thus providing useful clinical and epidemiologic information. The role of autopsy still remains unchanged despite the decline in autopsy rates. During the last few decades, several factors such as time constraints, attitudes (of clinicians, pathologists, families, or administrators), and costs (professional and overheads) have negatively influenced the autopsy rate, leading to the lack of substantial information [4]. In 1996, Schwartz and Herman [5] highlighted the importance of autopsy, particularly in emerging and re-emerging infectious diseases.

During the recent outbreak of the Middle East respiratory syndrome (MERS), the value of this investigation tool was emphasized. The first autopsy report was published in 2016, two years after it had been performed in 2014, while the infection had started in 2012. Analyzing the data reported by $\mathrm{Ng}$ et al. [6], the autopsy provided unprecedented, clinically-relevant insights about how this unknown infection had progressed, challenging previously accepted ideas about MERS. Autopsy provides critical information regarding emerging or unknown infectious diseases $[5,7]$.

\section{Methods}

\subsection{Database Search Terms and Timeline}

We performed a literature search on online resources (PubMed database) from 1 January 2019 to 28 April 2020, using the following key search terms: "COVID-19", "nCov 19", and "Sars Cov 2".

\subsection{Study Selection}

We retrieved 9709 articles; by excluding all duplicated articles, additional criteria were then applied: articles or abstract in English and articles containing one of the following words: "“death", "died", "comorbidity", "cause of death", "biopsy", "autopsy", or "pathological". To identify further studies that met the inclusion criteria, the references of the selected articles were also reviewed. Articles were excluded if they did not meet the inclusion criteria outlined previously. Figure 1 summarizes the data obtained after our PubMed literature search. 


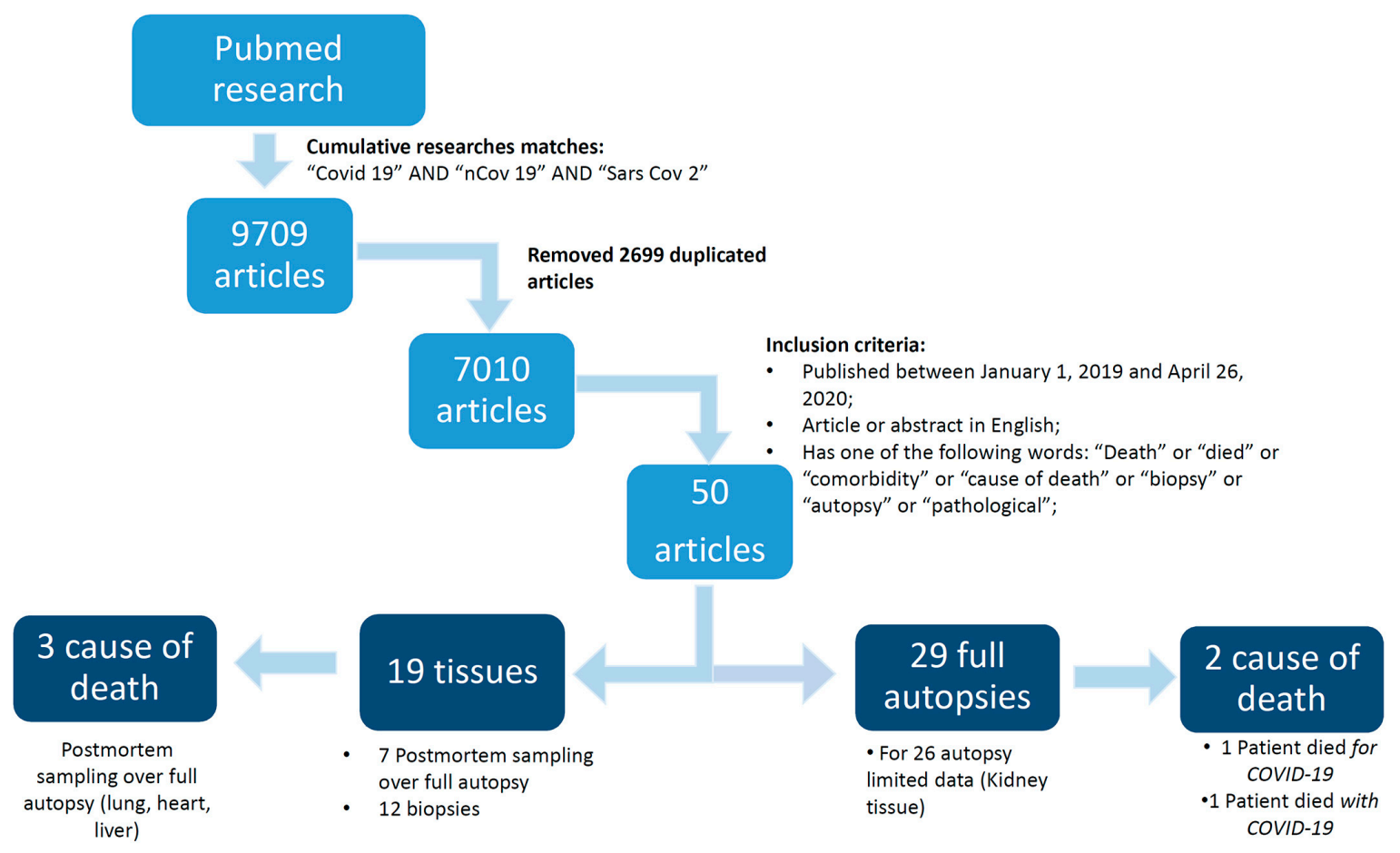

Figure 1. The search strategy used for literature review.

\section{Results}

A total of 50 articles met the inclusion criteria out of the 7010 articles found [8-17]. The main characteristics of each selected article are summarized in Table 1.

Analyzing Table 1, only in seven papers had the analysis of tissue samples been performed.

Tian et al. [9] analyzed two patients infected by COVID-19 with lung cancer. Two lung biopsies were performed before COVID-19 diagnosis and showed the following: alveolar edema and proteinaceous exudates, vascular congestion but patchy and mild inflammatory infiltration, focally fibrin clusters mixed with mononuclear inflammatory cells, and multinucleated giant cells were noted in the airspaces; no significant neutrophil infiltration was present in lung tissue, pneumocyte hyperplasia and interstitial thickening were also observed, indicating an ongoing reparative process. Suspected viral inclusions were also noted in some of these cells. At the time of manuscript preparation (submission on the 18 of February 2020), they have remarked that no full autopsy was performed on patients with COVID-19. At the same time, no data on lung biopsies for the COVID-19 infection were collected. Moreover, the authors highlighted the importance of the autopsy specimens to perform routine histopathological and immunohistochemical analyses combined with other investigations, such as RT-PCR, to understand better the mechanism of action of SARS-CoV-2 [9].

Respiratory tract involvement is further described by $\mathrm{Xu}$ et al. [11], who described for the first time the pathological findings of COVID-19 associated with acute respiratory distress syndrome. The authors collected post-mortem specimens from lung, heart, and liver tissues. Specimens from lungs showed the following: desquamation of pneumocytes and hyaline membrane formation, indicating acute respiratory distress syndrome, with interstitial mononuclear inflammatory infiltrates, dominated by lymphocytes. Pneumocytes were characterized by viral cytopathic-like changes. In their conclusions, the authors highlighted that their clinical and pathological findings in the reported case of COVID-19 were not only useful to identify the cause of death, but also to provide new insights into the pathogenesis of SARS-CoV-2-related pneumonia. Moreover, these findings could be beneficial for physicians to formulate a suitable therapeutic strategy for similar severe patients, reducing mortality. 
Table 1. The main characteristics of the selected studies are summarized in the table. All studies were published in peer-reviewed journals indexed in PubMed in 2020.

\begin{tabular}{|c|c|c|c|c|c|c|c|}
\hline Author & $\begin{array}{l}\text { Number of } \\
\text { Patients }\end{array}$ & $\begin{array}{l}\text { Patients } \\
\text { Who Died }\end{array}$ & $\begin{array}{l}\text { Average Age } \\
\text { (Years) }\end{array}$ & Comorbidities & Severe Complications & $\begin{array}{l}\text { Tissues } \\
\text { (Biopsy or } \\
\text { Autopsy } \\
\text { Samples) }\end{array}$ & Autopsy \\
\hline Peng Y. D. et al. [8] & 112 & 17 & $\begin{array}{l}\text { Data not } \\
\text { available }\end{array}$ & $\begin{array}{l}\text { Coronary heart disease (CHD) }(100 \%), \\
\text { BMI }>25(88.24 \%)\end{array}$ & Data not available & 0 & 0 \\
\hline Tian S. et al. [9] & 2 & 2 & 78.5 & $\begin{array}{c}\text { lung cancer }(100 \%) \text {, hypertension }(100 \%), \\
\text { diabetes }(50 \%)\end{array}$ & Respiratory failure, coma, heart failure & $\begin{array}{c}2 \\
\text { (biopsy) }\end{array}$ & 0 \\
\hline Guan W. J. et al. [10] & 1099 & 15 & 47 & $\begin{array}{c}\text { Hypertension }(15 \%) \text {, diabetes }(7.4 \%), \\
\text { CHD }(2.5 \%), \text { HCV }(2.1 \%) \text {, chronic } \\
\text { obstructive pulmonary disease (COPD) } \\
(1.1 \%) \text {, cancer }(0.9 \%)\end{array}$ & $\begin{array}{l}\text { Septic shock, acute respiratory distress } \\
\text { syndrome (ARDS), kidney failure }\end{array}$ & 0 & 0 \\
\hline Xu Z. et al. [11] & 1 & 1 & 50 & Data not available & Respiratory failure, ARDS & $\begin{array}{c}1 \\
\text { (autoptic } \\
\text { sample) }\end{array}$ & 0 \\
\hline Kui K. et al. [12] & 137 & 16 & 57 & $\begin{array}{c}\text { Hypertension (9.5\%), diabetes }(10.2 \%) \\
\text { CHD }(7.3 \%) \text {, COPD }(1.5 \%) \text {, cancer }(1.5 \%)\end{array}$ & Data not available & 0 & 0 \\
\hline Wang W. et al. [13] & 571 & 17 & 73 & $\begin{array}{c}\text { Hypertension }(41.2 \%) \text {, diabetes }(23.5 \%), \\
\text { CHD }(17.6 \%) \text {, stroke }(17.6 \%), \text { COPD } \\
(11.7 \%), \text { kidney failure }(11.7 \%), \\
\text { Parkinson }(11.7 \%), \text { cancer }(5.9 \%) \\
\text { cirrhosis }(5.9 \%)\end{array}$ & Data not available & 0 & 0 \\
\hline Yang X. et al. [14] & 201 & 32 & 59.7 & $\begin{array}{l}\text { CHD }(9 \%), \text { COPD }(6 \%), \text { diabetes }(22 \%), \\
\text { cancer }(3 \%), \text { stroke }(22 \%)\end{array}$ & $\begin{array}{l}\text { ARDS, kidney failure, heart failure, liver } \\
\text { failure }\end{array}$ & 0 & 0 \\
\hline Huang C. et al. [15] & 41 & 6 & 49 & $\begin{array}{l}\text { Diabetes (20\%), hypertension (15\%), } \\
\text { cardiovascular disease (CVD) (15\%) }\end{array}$ & $\begin{array}{l}\text { Respiratory distress syndrome }(29 \%), \\
\text { RNAaemia (15\%), acute cardiac injury } \\
(12 \%) \text {, secondary infection }(10 \%)\end{array}$ & 0 & 0 \\
\hline Wang D. et al. [16] & 138 & 6 & 56 & $\begin{array}{l}\text { Hypertension }(31.2 \%) \text {, diabetes }(10.1 \%), \\
\text { CVD }(14.5 \%), \text { Cancer }(7.2 \%)\end{array}$ & ARDS, arrhythmia, shock. & 0 & 0 \\
\hline Chen N. et al. [17] & 99 & 11 & 55.5 & $\begin{array}{c}\text { CVD and cerebrovascular diseases }(40 \%), \\
\text { digestive system disease }(11 \%), \\
\text { endocrine system disease }(13 \%), \text { cancer } \\
(1 \%), \text { nervous system disease }(1 \%), \\
\text { respiratory system disease }(1 \%)\end{array}$ & $\begin{array}{c}\text { ARDS (17\%), kidney failure }(3 \%), \\
\text { respiratory failure }(8 \%) \text {, Septic shock } \\
(4 \%)\end{array}$ & 0 & 0 \\
\hline
\end{tabular}


Table 1. Cont.

\begin{tabular}{|c|c|c|c|c|c|c|c|}
\hline Author & $\begin{array}{l}\text { Number of } \\
\text { Patients }\end{array}$ & $\begin{array}{l}\text { Patients } \\
\text { Who Died }\end{array}$ & $\begin{array}{l}\text { Average Age } \\
\text { (Years) }\end{array}$ & Comorbidities & Severe Complications & $\begin{array}{l}\text { Tissues } \\
\text { (Biopsy or } \\
\text { Autopsy } \\
\text { Samples) }\end{array}$ & Autopsy \\
\hline Zhang et al. [18] & 7 & 5 & 59 & Not indicated & $\begin{array}{l}\text { Acro-ischemia presentations including } \\
\text { finger/toe cyanosis, skin bulla and dry } \\
\text { gangrene }(100 \%) \text {, definite disseminated } \\
\text { intravascular coagulation (DIC) }(4(57 \%))\end{array}$ & 0 & 0 \\
\hline $\begin{array}{c}\text { Korean Society of } \\
\text { Infectious Diseases et al. } \\
\text { [19] }\end{array}$ & 54 & 54 & 75.5 & $\begin{array}{c}\text { CVD }(59.3 \%) \text {; diabetes mellitus (DM) } \\
(29.6 \%) \text {; neurological disease }(18.5 \%) ; \\
\text { lung disease }(13.0 \%) \text {; malignancy }(13.0 \%) ; \\
\text { psychologic disease }(13.0 \%) ; \text { renal } \\
\text { disease }(9.3 \%) \text {; hepatic disease }(3.7 \%) ; \\
\text { kidney transplant recipient }(1.9 \%)\end{array}$ & Data not available & 0 & 0 \\
\hline Yuan et al. [20] & 27 & 10 & 60 & $\begin{array}{l}\text { Hypertension (19\%); diabetes }(22 \%) \text {; } \\
\text { CVD (11\%); tumor (4\%); cerebral } \\
\text { infarction ( }(4 \%) \text {; chronic gastritis }(4 \%)\end{array}$ & ARDS (41\%) & 0 & 0 \\
\hline Guo et al. [21] & 187 & 43 & 58.5 & $\begin{array}{c}\text { Hypertension (32.6\%); CHD (11.2\%); } \\
\text { cardiomyopathy }(8(4.3 \%)) \text {; diabetes } \\
(15.0 \%) ; \text { COPD }(2.1) \text {; malignant } \\
\text { neoplasm (7.0\%); chronic kidney disease } \\
\text { (CKD) }(3.2 \%)\end{array}$ & $\begin{array}{c}\text { ARDS }(24.6 \%) \text {, malignant arrhythmias } \\
\quad(5.9 \%) \text { including ventricular } \\
\text { tachycardia/ventricular fibrillation, acute } \\
\text { coagulopathy }(34.1 \%) \text {, acute liver injury } \\
(15.4 \%) \text { and acute kidney injury }(14.6 \%)\end{array}$ & 0 & 0 \\
\hline Yang et al. [22] & 25 & 5 & 60.2 & $\begin{array}{l}\text { Hypertension }(15.4 \%) \text {; diabetes }(7.7 \%) ; \\
\text { COPD }(38.5 \%) ; \text { CHD }(30.8 \%)\end{array}$ & Data not available & 0 & 0 \\
\hline Chen et al. [23] & 274 & 113 & 62 & $\begin{array}{l}\text { Hypertension }(34 \%) \text {; diabetes }(17 \%) ; \\
\text { CVD }(8 \%) ; \text { CHD (<1\%); COPD }(7 \%) ; \\
\text { malignancy }(3 \%) \text {; hepatitis B (4\%); } \\
\text { cerebrovascular disease (1\%); CKD }(1 \%) ; \\
\text { gastrointestinal diseases }(1 \%) \text {; metabolic } \\
\text { arthritis }(1 \%) ; \text { autoimmune disease }(1 \%)\end{array}$ & $\begin{array}{l}\text { ARDS }(72 \%) \text {, type I respiratory failure } \\
(27 \%) \text {, acute cardiac injury }(44 \%), \text { heart } \\
\text { failure }(24 \%) \text {, hypoxic encephalopathy } \\
(9 \%), \text { sepsis }(65 \%) \text {, acidosis }(12 \%), \\
\text { alkalosis }(28 \%), \text { acute kidney injury } \\
(11 \%), \text { disseminated intravascular } \\
\text { coagulation }(8 \%) \text {, hyperkalemia }(23 \%), \\
\text { shock }(17 \%), \text { acute liver injury }(5 \%), \\
\text { gastrointestinal bleeding }(<1 \%) .\end{array}$ & 0 & 0 \\
\hline
\end{tabular}


Table 1. Cont.

\begin{tabular}{|c|c|c|c|c|c|c|c|}
\hline Author & $\begin{array}{l}\text { Number of } \\
\text { Patients }\end{array}$ & $\begin{array}{l}\text { Patients } \\
\text { Who Died }\end{array}$ & $\begin{array}{l}\text { Average Age } \\
\text { (Years) }\end{array}$ & Comorbidities & Severe Complications & $\begin{array}{l}\text { Tissues } \\
\text { (Biopsy or } \\
\text { Autopsy } \\
\text { Samples) }\end{array}$ & Autopsy \\
\hline Zhuo et al. [24] & 191 & 54 & $\begin{array}{l}\text { Data not } \\
\text { available }\end{array}$ & Data not available & $\begin{array}{c}\text { Sepsis }(59 \%), \text { respiratory failure }(54 \%), \\
\text { ARDS }(31 \%) \text {, heart failure }(23 \%) \text {, septic } \\
\text { shock }(20 \%) \text {, coagulopathy }(19 \%) \text {, acute } \\
\text { cardiac injury }(17 \%) \text {, acute kidney injury } \\
(15 \%) \text {, secondary infection }(15 \%), \\
\text { hypoproteinemia }(12 \%) \text {, acidosis }(9 \%)\end{array}$ & 0 & 0 \\
\hline Deng et al. [25] & 225 & 109 & 69 & $\begin{array}{c}\text { Hypertension (36.7\%); lung disease } \\
(20.2 \%) \text {; diabetes }(15.6 \%) \text {; heart disease } \\
(11.9 \%) \text {; malignancy }(5.5 \%) \text {; others } \\
(28.4 \%)\end{array}$ & $\begin{array}{c}\text { ARDS }(7.6 \%) \text {, acute cardiac injury }(0.8 \%), \\
\text { acute kidney injury }(<1 \%) \text {, shock }(<1 \%), \\
\text { and disseminated intravascular } \\
\text { coagulation (DIC) }(<1 \%)\end{array}$ & 0 & 0 \\
\hline $\begin{array}{l}\text { Rodriguez-Morales et al. } \\
\text { [26] }\end{array}$ & 2874 & 632 & 51.97 & $\begin{array}{c}\text { Hypertension }(18.6 \%) \text {; CVD }(14.4 \%) ; \\
\text { diabetes }(11.9 \%) \text {; COPD }(1.8 \%) ; \\
\text { malignancies }(2.5 \%) ; \text { chronic liver } \\
\text { disease (CLD) }(3.0 \%)\end{array}$ & $\begin{array}{c}\text { 20.3\% who required ICU: ARDS ( } 32.8 \%) \\
\text { cardiac injury }(13.0 \%), \text { acute kidney } \\
\text { injury }(7.9 \%) \text {, shock }(6.2 \%) \text {, Secondary } \\
\text { infections }(5.6 \%) .\end{array}$ & 0 & 0 \\
\hline Guan et al. [27] & 1590 & 50 & 48.9 & $\begin{array}{c}\text { Hypertension }(16.7 \%) \text {; CVD }(53.7 \%) \text {, } \\
\text { cerebrovascular disease }(1.9 \%) \text {, diabetes } \\
(8.2 \%) \text {, hepatitis B }(1.8 \%), \text { COPD }(1.5 \%), \\
\text { CKD }(1.3 \%) \text {, malignncy }(1.1 \%)\end{array}$ & Data not available & 0 & 0 \\
\hline Bhatraju et al. [28] & 24 & 12 & 64 & $\begin{array}{l}\text { Asthma }(14 \%), \text { CKD }(21 \%) \text {, COPD }(4 \%), \\
\text { tobacco smoker }(22 \%) \text {, diabetes }(58 \%),\end{array}$ & Data not available & 0 & 0 \\
\hline Zhang et al. [29] & 28 & 8 & 65 & $\begin{array}{c}\text { Cancer }(100 \%) \text {, diabetes } 4(14.3 \%) \text {, COPD } \\
1(3.6 \%)\end{array}$ & $\begin{array}{c}\text { ARDS } 8(28.6 \%) \text {, septic shock } 1(3.6 \%), \\
\text { suspected pulmonary embolism } 2(7.1 \%), \\
\text { AMI } 1(3.6 \%)\end{array}$ & 0 & 0 \\
\hline Lescure et al. [30] & 5 & 1 & 47 & $\begin{array}{c}\text { Hypertension } 1(20 \%) \text {, cancer } 1(20 \%), \\
\text { gout } 1(20 \%)\end{array}$ & Data not available & 0 & 0 \\
\hline Wu et al. [31] & 201 & 44 & 51 & $\begin{array}{c}\text { Hypertension }(19.4 \%) \text { diabetes }(10.9 \%) \\
\text { CVD }(4.0 \%) \text { liver disease }(3.5 \%) \text { nervous } \\
\text { system disease }(3.5 \%) \text { chronic lung } \\
\text { disease }(2.5 \%) \text { CKD }(1.0 \%) \text { endocrine } \\
\text { system disease }(1.0 \%) \text { tumor }(0.5 \%)\end{array}$ & Data not available & 0 & 0 \\
\hline
\end{tabular}


Table 1. Cont.

\begin{tabular}{|c|c|c|c|c|c|c|c|}
\hline Author & $\begin{array}{l}\text { Number of } \\
\text { Patients }\end{array}$ & $\begin{array}{l}\text { Patients } \\
\text { Who Died }\end{array}$ & $\begin{array}{l}\text { Average Age } \\
\text { (Years) }\end{array}$ & Comorbidities & Severe Complications & $\begin{array}{l}\text { Tissues } \\
\text { (Biopsy or } \\
\text { Autopsy } \\
\text { Samples) }\end{array}$ & Autopsy \\
\hline Grasselli et al. [32] & 1591 & 405 & 63 & $\begin{array}{c}\text { Hypertension (49\%); CVD (21\%); } \\
\text { hypercholesterolemia (18\%); DM (17); } \\
\text { malignancy ( } 8 \%) \text {; COPD }(4 \%) \text {; CKD }(3 \%) \text {; } \\
\text { CLD }(3 \%) \text {; other }(20 \%)\end{array}$ & Data not available & 0 & 0 \\
\hline Bobin et al. [33] & 10 & 4 & 68.4 & $\begin{array}{c}\text { Fracture }(100 \%) \text {; hypertension }(40 \%) ; \\
\text { diabetes }(30 \%) ; \operatorname{COPD}(10 \%) ; \\
\text { osteoporosis }(30 \%) ; \text { CHD }(10 \%) \text {; cirrhosis } \\
(10 \%) \text {, alzheimer disease }(10 \%) ; \text { brain } \\
\text { injury }(10 \%)\end{array}$ & Data not available & 0 & 0 \\
\hline Chen et al. [34] & 203 & 26 & 54 & $\begin{array}{c}\text { Hypertension (21.2\%); diabetes (7.9\%); } \\
\text { CVD (7.9\%); cerebrovascular disease } \\
(4.4 \%) ; \text { malignancy }(3,4 \%) ; \text { CLD }(3.9 \%) ; \\
\text { CKD } \\
(8(3.9 \%)) ; \text { COPD }(8(3.9 \%)) ; \text { Tuberculosis } \\
(4(2.0 \%)) ; \operatorname{HIV~}(2(0.1 \%))\end{array}$ & $\begin{array}{l}\text { Cause of Death: ARDS (14\%); ARDS with } \\
\text { MOD ( } 22 \%) \text {; sepsis/Shock }(4 \%) \text {; heart } \\
\text { failure }(2 \%) \text {; myocardial infarction }(6 \%) \text {; } \\
\text { tumor }(4 \%) \text {; intestinal bleeding }(2 \%)\end{array}$ & 0 & 0 \\
\hline Li et al. [35] & 25 & 25 & 73 & $\begin{array}{c}\text { Hypertension }(64 \%) \text {; diabetes }(40 \%) ; \\
\text { heart diseases }(32 \%) ; \text { kidney diseases } \\
(20 \%) \text {; cerebral infarction }(16 \%) ; \\
\text { COPD ( } 8 \%) \text {; malignant tumors }(8 \%) ; \\
\text { acute pancreatitis }(4 \%)\end{array}$ & Respiratory failure (100\%) & 0 & 0 \\
\hline Cao et al. [36] & 102 & 17 & 54 & $\begin{array}{c}\text { Hypertension }(27.5 \%) \text {; diabetes }(10.8 \%) ; \\
\text { cerebrovascular disease }(5.9 \%) ; \text { CVD } \\
(4.9 \%) \text {; respiratory diseases }(9.8 \%) ; \\
\text { malignancy }(3.9 \%) \text {; CKD }(3.9 \%) ; \text { CLD } \\
(2.0 \%) ;\end{array}$ & $\begin{array}{c}\text { Shock (9.8\%); ARDS (19.6\%); acute } \\
\text { infection (16.7\%); acute cardiac injury } \\
\text { (14.7\%); arrhythmia (17.6\%); acute kidney } \\
\text { injury (19.6\%); acute liver injury (33.3\%); } \\
\text { lymphopenia (76.5\%); Cause of Death: } \\
\text { multiple organ dysfunction syndrome } \\
\text { (MODS) }(58.8 \%) ; \text { ARDS (5.9\%); cardiac } \\
\text { arrest (23.5\%); respiratory failure (11.8\%) }\end{array}$ & 0 & 0 \\
\hline Wang et al. [37] & 339 & 65 & 71 & $\begin{array}{c}\text { Hypertension }(40.8 \%) \text {, diabetes }(16.0 \%) \text {, } \\
\text { CVD }(15.7 \%)\end{array}$ & $\begin{array}{c}\text { Lymphocytopenia }(63.2 \%) \text {, bacterial } \\
\text { infection }(42.8 \%) \text {, liver enzyme } \\
\text { abnormalities } \\
(28.7 \%) \text {, acute respiratory distress } \\
\text { syndrome }(21.0 \%)\end{array}$ & 0 & 0 \\
\hline
\end{tabular}


Table 1. Cont.

\begin{tabular}{|c|c|c|c|c|c|c|c|}
\hline Author & $\begin{array}{l}\text { Number of } \\
\text { Patients }\end{array}$ & $\begin{array}{l}\text { Patients } \\
\text { Who Died }\end{array}$ & $\begin{array}{l}\text { Average Age } \\
\text { (Years) }\end{array}$ & Comorbidities & Severe Complications & $\begin{array}{l}\text { Tissues } \\
\text { (Biopsy or } \\
\text { Autopsy } \\
\text { Samples) }\end{array}$ & Autopsy \\
\hline \multirow[t]{2}{*}{ Barton et al. [38] } & \multirow[t]{2}{*}{2} & \multirow[t]{2}{*}{2} & 77 & $\begin{array}{l}\text { Hypertension, splenectomy, } \\
\text { cholelithiasis, osteoarthritis }\end{array}$ & $\begin{array}{c}\text { Cause of Death: COVID-19 } \\
\text { (ARDS—diffuse alveolar damage (DAD)) }\end{array}$ & \multirow[t]{2}{*}{0} & \multirow[t]{2}{*}{2} \\
\hline & & & 42 & $\begin{array}{l}\text { Myotonic, } \\
\text { muscular dystrophy }\end{array}$ & $\begin{array}{l}\text { Complications of hepatic cirrhosis } \\
\text { (aspiration acute bacterial } \\
\text { bronchopneumonia) }\end{array}$ & & \\
\hline Huang et al. [39] & 2 & 2 & 54.5 & Transplantation $(100 \%)$ & $\begin{array}{c}\text { Nosocomial bacterial infection }(100 \%) \text {; } \\
\text { respiratory organ failure }(100 \%) \text {; kidney } \\
\text { organ failure (100\%); heart organ failure } \\
(50 \%)\end{array}$ & 0 & 0 \\
\hline Ling et al. [40] & 8 & 1 & 64.5 & Data not available & $\begin{array}{l}\text { Respiratory failure }(75 \%) \text {; kidney failure } \\
\qquad(25 \%)\end{array}$ & 0 & 0 \\
\hline Cheng et al. [41] & 701 & 113 & 63 & $\begin{array}{c}\text { Any comorbidity }(42.6 \%) ; \text { CKD }(2.0 \%) ; \\
\text { COPD }(1.9 \%) ; \text { hypertension }(33.4 \%) ; \\
\text { diabetes }(14.3 \%) ; \text { tumor }(4.6 \%)\end{array}$ & Acute kidney injury (5.1\%) & 0 & 0 \\
\hline Du et al. [42] & 179 & 21 & 57.6 & $\begin{array}{c}\text { Hypertension (32.4\%); CVD or } \\
\text { cerebrovascular diseases (16.2\%); } \\
\text { diabetes (18.4\%); chronic digestive } \\
\text { disorders (11.7\%); tuberculosis }(4.5 \%) \text {; } \\
\text { chronic hepatic or renal insufficiency } \\
\text { (2.2\%); Peripheral vascular disease } \\
(2.2 \%) ; \text { malignancy }(2.2 \%)\end{array}$ & Data not available & 0 & 0 \\
\hline Barrasa et al. [43] & 48 & 14 & 63 & $\begin{array}{c}\text { Obesity (48\%); arterial } \\
\text { hypertension ( } 44 \%) \text {; COPD (37\%); }\end{array}$ & Hypoxemic respiratory failure $(100 \%)$ & 0 & 0 \\
\hline Lovell et al. [44] & 101 & 75 & 82 & $\begin{array}{l}\text { Hypertension (54\%); diabetes (36\%); } \\
\text { dementia (31\%); cancer ( } 25 \%) \text {; COPD } \\
\text { (22\%); renal failure ( } 21 \%) \text {; congestive } \\
\text { heart failure }(18 \%) \text {; stroke / neurological } \\
\text { disorder }(12 \%) \text {; peripheral vascular } \\
\text { disorder }(4 \%) \text {; liver disease }(2 \%) ;\end{array}$ & Data not available & 0 & 0 \\
\hline Wang et al. [45] & 80 & 1 & 39 & $\begin{array}{c}\text { Hypertension }(12.5 \%) \text {; diabetes }(1.25 \%) ; \\
\text { CVD ( } 2.5 \%) \text {; cerebrovascular disease } \\
(1.25 \%) \text {; COPD } 1(1.25 \%) \text {; renal disease } \\
(3.75 \%) \text {; liver disease }(2.5 \%)\end{array}$ & Data not available & 0 & 0 \\
\hline
\end{tabular}


Table 1. Cont.

\begin{tabular}{|c|c|c|c|c|c|c|c|}
\hline Author & $\begin{array}{l}\text { Number of } \\
\text { Patients }\end{array}$ & $\begin{array}{l}\text { Patients } \\
\text { Who Died }\end{array}$ & $\begin{array}{l}\text { Average Age } \\
\text { (Years) }\end{array}$ & Comorbidities & Severe Complications & $\begin{array}{l}\text { Tissues } \\
\text { (Biopsy or } \\
\text { Autopsy } \\
\text { Samples) }\end{array}$ & Autopsy \\
\hline Zhang et al. [46] & 221 & 12 & 55 & $\begin{array}{c}\text { Hypertension }(24.4 \%) \text {, diabetes }(10.0 \%), \\
\text { CVD }(10.0 \%) \text {, cerebrovascular disease } \\
(6.8 \%), \text { COPD }(2.7 \%), \\
\text { CKD }(2.7 \%), \text { CLD }(3.2 \%) \text {, malignancy } \\
(4.1 \%), \text { immunosuppression treatment } \\
(1.4 \%)\end{array}$ & $\begin{array}{c}\text { ARDS (21.7); arrhythmia (10.9); acute } \\
\text { cardiac injury (7.7); shock (6.8); AKI (4.5) }\end{array}$ & 0 & 0 \\
\hline Magro et al. [47] & 5 & 2 & 54.6 & $\begin{array}{l}\text { Coronary artery disease, diabetes } \\
\text { mellitus, heart failure, hepatitis } C \text { virus } \\
\text { infection, end-stage renal disease, obesity } \\
(n=2) \text {, and pre-diabetes, }\end{array}$ & $\begin{array}{l}\text { Respiratory failure }(n=5) \text {; purpuric skin } \\
\qquad \text { rash }(n=3)\end{array}$ & $\begin{array}{l}3 \text { cases skin } \\
\text { biopsies }\end{array}$ & $\begin{array}{l}2 \text { Cases, } \\
\text { limited } \\
\text { autopsy }\end{array}$ \\
\hline Pereira et al. [48] & 90 & 16 & 57 & $\begin{array}{c}\text { HTN }(64 \%) \text {; diabetes }(46 \%) \text {; CKD }(63 \%) ; \\
\text { dialysis }(6 \%) \text {; chronic lung disease }(19 \%) \text {; } \\
\text { HIV }(1 \%) \text {; active cancer }(3 \%) ; \\
\text { BMI > } 40 \mathrm{Kg} / \mathrm{m}^{2}(6 \%)\end{array}$ & Data not available & 0 & 0 \\
\hline Li et al. [49] & 658 & 64 & 47 & $\begin{array}{c}\text { Cerebrovascular disease }(8 \%) \text {; coronary } \\
\text { heart disease }(8.9 \%) \text {; heart failure }(1.21 \%) \text {; } \\
\text { diabetes }(19.6 \%) \text {;ypertension }(33.4 \%) ; \\
\text { digestive disorder }(13.22 \%) \text {; COPD } \\
(2.88 \%) ; \text { cancer }(2.58 \%) ; \text { CKD }(2.73 \%) \text {; } \\
\text { hepatitis }(1.06 \%)\end{array}$ & $\begin{array}{c}\text { Ketosis (6.38\%); acute liver injury } \\
(5.92 \%) ; \text { septic shock (5.31\%); ARDS } \\
(14.43 \%) \text {; diabetic ketoacidosis (DKA) } \\
(0.4 \%) ; \text { acidosis }(4.55 \%)\end{array}$ & 0 & 0 \\
\hline Yang et al. [50] & 92 & 91 & 69.8 & $\begin{array}{c}\text { Hypertension (55.43\%); heart disease } \\
(17.39 \%) ; \text { cerebrovascular }(10.8 \%) ; \\
\text { malignancy (4.34\%); CLD (3.26\%); CKD } \\
(2.17 \%) ; \text { COPD }(1 \%)\end{array}$ & $\begin{array}{c}\text { Cause of death: ARDS }(79.34 \%) \text {, septic } \\
\text { shock }(7.6 \%) \text {, myocardial infarction } \\
(6.52 \%), \text { heart failure }(2.17 \%) \text {, MODS } \\
(2.17 \%)\end{array}$ & 0 & 0 \\
\hline Cai et al. [51] & 7 & 3 & 61 & $\begin{array}{l}\text { COPD }(28.5 \%) \text {; CVD }(42.8 \%) \text {; interstitial } \\
\text { lung disease }(14.28 \%) \text {; hyperlipidemia } \\
(14.28 \%) ; \text { Malignancy }(100 \%)\end{array}$ & Cause of death: respiratory failure 3 & $\begin{array}{c}7 \\
\text { Biopsies }\end{array}$ & 0 \\
\hline Karami et al. [52] & 1 & 1 & 27 & No underlying disease & Data not available & 0 & 1 \\
\hline Tian et al. [53] & 4 & 4 & 73 & $\begin{array}{l}\text { Chronic lymphocytic leukemia (CLL), } \\
\text { cirrhosis, hypertension, diabetes, } \\
\text { and renal transplantation }\end{array}$ & Data not available & $\begin{array}{c}4 \\
\text { (Autopsy } \\
\text { samples) }\end{array}$ & 0 \\
\hline Fabre et al. [54] & 1 & 1 & 45 & Obesity (BMI 40.4), hypertension & Pulmonary Embolism & 0 & 0 \\
\hline
\end{tabular}


Table 1. Cont.

\begin{tabular}{|c|c|c|c|c|c|c|c|}
\hline Author & $\begin{array}{l}\text { Number of } \\
\text { Patients }\end{array}$ & $\begin{array}{l}\text { Patients } \\
\text { Who Died }\end{array}$ & $\begin{array}{l}\text { Average Age } \\
\text { (Years) }\end{array}$ & Comorbidities & Severe Complications & $\begin{array}{l}\text { Tissues } \\
\text { (Biopsy or } \\
\text { Autopsy } \\
\text { Samples) }\end{array}$ & Autopsy \\
\hline Su et al. [55] & 26 & 26 & 69 & $\begin{array}{l}\text { History of hypertension or diabetes or } \\
\text { both }(42.3 \%)\end{array}$ & Data not available & $\begin{array}{l}26 \\
\text { (Kidney } \\
\text { autopsy } \\
\text { samples) }\end{array}$ & 0 \\
\hline Edrada et al. [56] & 2 & 2 & 42 & Data not available & Data not available & 0 & 0 \\
\hline Zhang et al. [57] & 343 & 13 & 62 & $\begin{array}{c}\text { Diabetes }(13.7 \%) \text {, hypertension }(22.15 \%) \text {, } \\
\text { CHD }(5.5 \%) \text {, COPD }(2.33 \%) \text {, cancer } \\
(2.62 \%) \text {, stroke history }(2.33 \%) \text {, CLD } \\
(1.74 \%)\end{array}$ & Data not available & 0 & 0 \\
\hline
\end{tabular}


Barton et al. [38] described the findings of the two complete autopsies performed on COVID-19 patients. It is important to note that even if the macroscopic investigation was performed on several organs, the histological and immunohistochemical examinations were carried out on lung specimens. In the first case, both microscopic investigations reported the presence of thrombi within a few small pulmonary artery branches. Congestion of alveolar septal capillaries and edema fluid within the airspaces were also reported. For these reasons, the cause of death was listed as COVID-19. Conversely, in the second case there was no evidence of diffuse alveolar damage (DAD), even if immunohistochemistry showed similar findings to the other case. Therefore, considering the presence of other comorbidities, the cause of death was listed as "complications of hepatic cirrhosis", listing the COVID-19 infection under other significant conditions.

Magro et al. [47] examined lung and cutaneous tissues sampled from five patients with COVID-19 infection and severe respiratory failure, three of whom also had features consistent with a systemic procoagulant state. They performed histologic and immunohistochemical investigations, defining a pattern of cutaneous and pulmonary pathology involving microvascular injury and thrombosis.

Cai et al. [51] reported seven biopsies collected from COVID-19 patients during the perioperative period of lung resection. The histopathological investigation was performed to obtain information about the lung tumor. Interstitial inflammation with plasma cell and macrophage infiltration was detected.

Karami et al. [52] described the first case of pregnancy mortality due to COVID-19. A partial autopsy was performed; indeed, the authors reported that the patient underwent lung autopsy. The histologic investigation highlighted alveolar spaces with focal hyaline membrane, pneumocyte proliferation, and metaplastic changes. Moreover, many inflammatory cells were found. Nevertheless, the authors reported that these findings were not enough to define the exact cause of death. Moreover, they said that these difficulties can be related to the absence of published evidence on COVID-19, especially in pregnant patients.

Tian et al. [53] reported the postmortem investigations of core biopsies of lung, liver, and heart in four patients who died of COVID-19 pneumonia. Even if several important findings were described, the absence of complete autopsies did not provide all the necessary information.

Su et al. [55] focused their study on the autopsy renal tissue sampled in patients who died of COVID-19, reporting direct adverse effects of the virus on this tissue.

No evidence of biopsies or autopsy samples were described in the other selected papers.

In their different studies, Guan [10] and Peng [8] focused their analysis on the outcome of different cohorts of COVID-19 patients. To evaluate the outcome of infected patients, Guan et al. analyzed the medical records and compiled data for hospitalized patients and outpatients with laboratory-confirmed COVID-19 infection, reporting a mortality of $1.4 \%$.

Similarly, Peng et al. [8] analyzed the outcome of 112 COVID-19 patients affected by cardiovascular diseases. The authors reported that an overweight condition (BMI $>25 \mathrm{~kg} / \mathrm{m}^{2}$ ) was significantly higher in critical or not-survivor patients compared to survivors.

Other retrospective studies were discussed. Clinical data and therapeutic options were analyzed by Kui et al. [12] performing a retrospective study analyzing the clinical data from 137 COVID-19 infected patients. The authors identified significant clinical characteristics and corresponding treatment principles for the disease.

Another retrospective study was performed to define the main characteristics of this infection. In the 17 cases analyzed, Wang et al. [13] described that the first deaths were mainly among older people with comorbidities or a history of surgery before admission.

Chen et al. reported the retrospective analysis of 99 patients positive for COVID-19 infection. Interestingly, $51 \%$ of patients suffered from chronic diseases, including endocrine system diseases, cardiovascular and cerebrovascular diseases, digestive system diseases, malignant tumors, respiratory system diseases, and nervous system diseases [17].

In another important retrospective study, the authors concluded that during the COVID-19 infection, both pulmonary and systemic inflammation can lead to multi-organ dysfunction in patients 
at high risk [23]. In their retrospective analysis, Deng et al. [25] reported that more patients who died showed characteristics of advanced age and pre-existing comorbidities.

Lescure et al. [30] described the clinical history of five COVID-19 patients discussing the different outcomes of the infection.

All the other contributions focused their attention on different characteristics of the comorbidities related to patients hospitalized with COVID-19 infection.

Analyzing the report about the mortality cases of COVID-19 in the Republic of Korea, Yuan et al. [20] described the mortality rate as being significantly higher in the COVID-19 patients with several comorbidities such as hypertension, diabetes, and cardiac disease.

$\mathrm{Li}$ et al. [22] reported that COVID-19 is associated with poor prognosis for patients undergoing thoracic operations, especially for those with chronic obstructive pulmonary disease (COPD). Several studies were performed analyzing clinical, laboratory, imaging features, and outcomes of COVID-19 confirmed cases reporting the idea that the presence of comorbidities is an important factor in the mortality rate of the COVID-19 patients [26-29,31,32,36,37,39,40,43-46,48,49]. Yang et al. [50] described a retrospective study conducted on deceased patients, reporting the presence of ARDS, myocardial injury, liver injury, renal insufficiency, and multiple organ dysfunction syndrome (MODS) in COVID-19 patients. Du et al. [42] identified four risk factors: age $\geq 65$ years, preexisting concurrent cardiovascular or cerebrovascular diseases, $\mathrm{CD}^{+} \mathrm{CD}^{+} \mathrm{T}$ cells $\leq 75 \mathrm{cell} / \mu \mathrm{L}$, and cardiac troponin $\mathrm{I} \geq 0.05 \mathrm{ng} / \mathrm{mL}$; these last two factors were predictors for mortality of COVID-19 pneumonia patients. Moreover, other studies highlighted that males have a higher risk of death compared to females [34,35]. Furthermore, Li et al. [35] reported the importance of identifying critical patients quickly to improve their outcome. As described by Cheng et al. [41], acute kidney injury can be considered another important factor in patients' outcome.

Huang C. et al. [15] analyzed the data of 41 patients admitted to hospital with laboratory-confirmed COVID-19 infection. About 33\% of all patients had underlying diseases, such as diabetes, hypertension, and cardiovascular disease. Moreover, comparing the data of ICU patients with patients not treated in the ICU (102), they were older and with comorbidities.

The possible relationship between age, comorbidities, severity of respiratory distress, and death was then investigated by Yang et al. [14], who performed a full analysis of the electronic clinical medical records. Analyzing several aspects of deceased patients, they concluded that they were significantly older compared to survivors and with comorbidities and acute respiratory distress syndrome (ARDS).

Edrada et al. [56] reported two cases of COVID-19 patients; despite both patients being young adults with no significant past medical history, they had very different clinical courses. While one patient died after a few days of hospitalization, the other patient developed severe pneumonia and died. The different outcome is justified by the presence of several co-infections such as Influenza B, and Streptococcus pneumonia.

Zhang et al. [18] reported the existence of hypercoagulation status in critical COVID-19 patients, suggesting an anticoagulation therapy in similar patients. Fabre et al. [54] remarked that acute pulmonary embolism represents an important consequence in COVID-19 patients. The authors reported the case of a young woman presenting with severe pulmonary embolism, without any associated symptoms of infections, who died after 10 days of hospitalization. As reported by Guo et al. [21], myocardial injury represents an important factor associated with fatal outcomes of COVID-19. In the same way, Zhou et al. [24] reported that in COVID-19 patients, the presence of d-dimer levels greater than $1 \mu \mathrm{g} / \mathrm{mL}$ could help clinicians to identify patients with poor prognosis at an early stage. The higher d-dimer levels were also described by Bobin et al. [33]. As previously described, Magro et al. [47] tried to explain the pathogenesis of severe COVID-19 infection, describing an association with microvascular injury and thrombosis. Finally, in another recent report, d-dimer higher than $2.0 \mu \mathrm{g} / \mathrm{mL}$ on admission can be used as a predictor factor of in-hospital mortality in patients with COVID-19. Based on their data, d-dimer levels could be an early and helpful marker to improve the management of COVID-19 patients [57]. 


\section{Discussion}

The analysis of the main data from the selected studies concerns the complete analysis of 12,954 patients, of whom 2269 died (with a mortality rate of $17.52 \%$ ). The weighted mean age was 54.88 years. These data are strongly influenced by the selection criteria of the present study: indeed, retrospective studies are frequently performed in hospitalized patients. Laboratory confirmation of COVID-19 infection was obtained in all cases. Comorbidities were fully reported in 46 studies, and are summarized in Figure 2. The most common comorbidities were cardiovascular diseases (hypertension and coronary artery disease), metabolic disorders (diabetes, overweight, or obesity), respiratory disorders (chronic obstructive pulmonary disease), and cancer. Complications were reported in 30 articles. The most common reported complications were: acute respiratory distress syndrome (ARDS), acute kidney injury, cardiac injury, liver insufficiency, and septic shock.

\section{Comorbidities detected in patients who died with COVID-19 infection}

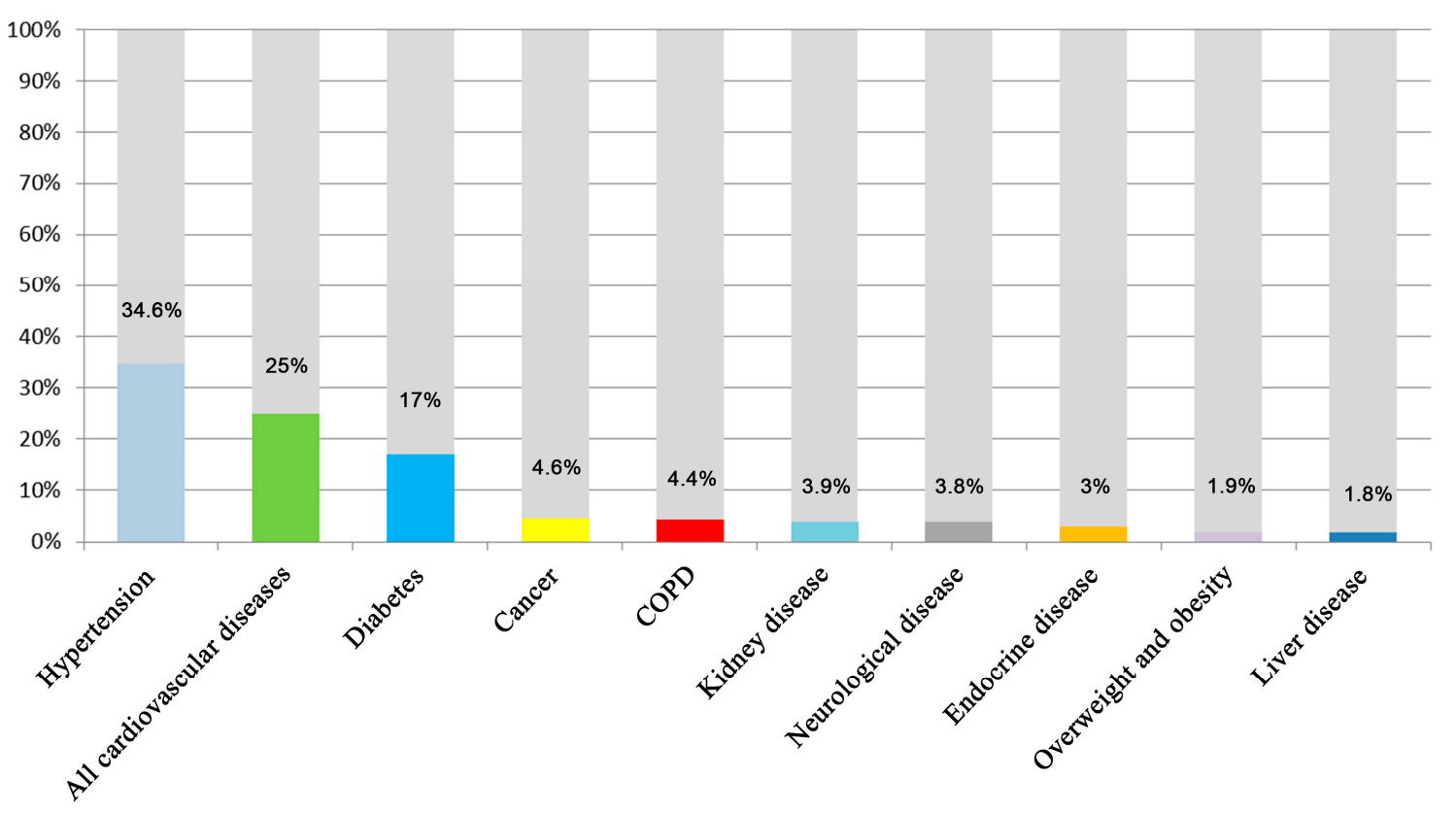

Figure 2. The histogram summarizes the comorbidity data in patients who died with COVID-19. Data are indicated in percentage for each comorbidity. Notably, percentage was obtained by indicating as the numerator the number of patients died who were positive for COVID-19 and affected by the specific comorbidity and, as the denominator, the total amount of patients who died and were positive for COVID-19.

Fifty articles were included in the present review, and only seven papers reported histological investigations. Nevertheless, only Barton et al. [38] described two complete autopsies. In the autopsy report of the first case, the cause of death was listed as COVID-19 in a patient with coronary artery disease, indicated under "other contributing factors". In the second case, the cause of death was listed as "complications of hepatic cirrhosis," with muscular dystrophy, aspiration pneumonia, and COVID-19 listed as other significant conditions.

In the light of this literature review, the lack of postmortem investigation did not allow a definition of the exact cause of death. In this way, it is very complex to define the exact pathways of this infection. Based on the few histopathological findings reported in the analyzed studies, it may be thought that this virus exerts adverse effects on the lung and kidney. There is scarce evidence about heart and brain tissues. Moreover, it seems to be a clear alteration of the coagulation system; prothrombotic activity with consequent thromboembolism was frequently described in COVID-19 patients. 
How can we estimate the real cause-specific mortality, including death rate, associated with COVID-19 when the cause of death is missing from the studies? How can we identify the additional care required for specific patient categories if there is still a lack of information about the real cause of death?

Such unsolved questions need to be addressed at the moment and should represent one of the goals of the scientific community.

Understanding the organ injury caused by COVID-19 and the underlying mechanisms could be a very helpful tool to optimize clinical management, helping clinicians to define a timely and effective treatment to reduce future mortality [58]. Indeed, after only about 1 month preliminary autopsy data (papers available in preprint version) suggested the importance of enhancing the host immune response against RNA viral infections. Recent reports about the pathological pathway of COVID-19 suggested that one of the most important consequences of this infection is the cytokine storm syndrome [15] that could be strictly linked with coagulopathy, generating acute pulmonary embolism caused by in-situ thrombosis $[59,60]$. In this way, a significant number of clinical trials are underway to define a useful therapy to attenuate cytokine storms [61].

For these reasons, it is important to also raise a political issue; although the World Health Organization suggested performing post-mortem examinations for those people who died with COVID-19 following recommended safety procedures [62], many Governments—including Italy—did not provide adequate tools to perform a sufficient number of autopsies. In Italy, despite the great action of our Government to face the epidemic, the Ministry of Health with a specific act (Circular of General Direction of Health Prevention) discourages the use of autopsy practice in COVID-19 deaths [63] and we are experiencing the incredible situation of having, unfortunately, thousands of deaths but almost no autopsies!

Considering that the COVID-19 infection is totally new, health care organizations and researchers have been publishing guidelines and recommendations to help health care providers proceed safely with various aspects of disease management and investigation $[64,65]$. Following these indications, it is possible to perform autopsies-that should be the way.

As recently argued by De Cock and Colleagues in the New England Journal of Medicine, despite the decline in autopsy rate a complete diagnostic autopsy remains the gold standard to determine why and how death happens, providing useful clinical information [66].

It is a fact that during the West Africa Ebola epidemic, in the Ebola virus disease (EVD) surveillance strategy, the RNA virus was isolated in body fluids days or months after the onset of the disease from any living or deceased individual who had, or had had, clinical symptoms compatible with EVD. Thanks to this procedure, it was possible to monitor the number of infected patients in order to recognize new sources of transmission and to control the epidemic phenomenon [67-73].

An equally effective method during yellow fever epidemic, was taking portions of post mortem liver parenchyma $[74,75]$.

In areas where systemic infections are prevalent, minimally invasive procedures with sampling of organs and tissues from cadavers can provide reliable data on specific causes of death [68].

Although collection of specimens from cadavers provided reliable data on specific causes of death and on detection of undocumented chains of transmission, only a full autopsy can investigate potential mechanisms of damage to organs or systems not readily accessible to biopsies, such as the central nervous system or cardiovascular system, leading to appropriate health-care strategies useful in the control of the disease [76].

Performing the histological and immunohistochemical investigations on the lung specimens collected during the autopsies of 8 subjects who died of SARS, the pathology of diffuse alveolar damage was described, providing the basis for therapeutic strategies [77].

Another important perspective that underlines the importance of autopsy is related to the development of vaccine candidates and new therapies for the prevention and treatment of lung disease caused by COVID-19 [4]. 
Moreover, about $7 \%-10 \%$ of the total amount of COVID- 19 infected patients require admission to an ICU $[10,14]$, and this percentage raises to about $35 \%$ among hospitalized patients with COVID-19 infection $[15,16]$.

Despite the introduction of more modern diagnostic techniques and of intensive and invasive monitoring, the number of missed major diagnoses in ICU deceased has not essentially changed over the past 20 to 30 years [78]; autopsies revealed ante mortem diagnostic errors or ante mortem unrecognized diagnoses in about $30 \%$ of cases $[79,80]$.

In a fascinating editorial published in 1970 in Chest, entitled "The Autopsy: Do We Still Need It?" [81], Edwards argued that despite the fact that the autopsy is the oldest method of medical investigation "... the current reports of autopsy findings serve to identify for current readers the nature of such conditions as a means of expanding the vistas of possible disease states". Since 1970, as highlighted above, nothing has changed about the diagnostic role of the autopsy and-despite the improvement and reinforcement of any method of study in every field of medicine and science-there is no objective justification for eliminating it.

We firmly believe that the answer to Edward's question in the 1970s is "yes, we still need the autopsy in 2020!" and we need it more nowadays, to answer a plethora of questions related to COVID-19 deaths.

As a scientific community, we are called on to face this global threat, and to defeat it with all available tools necessary, the new and the old ones, as the new and the old represent a proper union for continued progress in medicine.

This lesson, inherited from pioneers of medicine, should never be forgotten.

The time is now to shout out against this terrible lockdown of science: autopsy, autopsy, autopsy!

Author Contributions: Conceptualization, C.P., M.S., F.S., A.P. and G.L.V.; methodology, A.M., M.T., F.P., and P.M.; writing-original draft preparation, F.S., M.S., A.P., and C.P.; writing-review and editing, A.M., M.T., F.P., and P.M.; supervision, G.L.V. and C.P. All authors have read and agreed to the published version of the manuscript.

Funding: This research did not receive any specific grant from funding agencies in the public, commercial, or not-for-profit sectors.

Acknowledgments: The authors thank the Scientific Bureau of the University of Catania for language support.

Conflicts of Interest: The authors declare no conflict of interest.

\section{References}

1. Chan, J.F.W.; Yuan, S.; Kok, K.H.; To, K.K.W.; Chu, H.; Yang, J.; Xing, F.; Liu, J.; Yip, C.C.Y.; Poon, R.W.S.; et al. A familial cluster of pneumonia associated with the 2019 novel coronavirus indicating person-to-person transmission: a study of a family cluster. Lancet 2020. [CrossRef]

2. Messina, G.; Polito, R.; Monda, V.; Cipolloni, L.; Di Nunno, N.; Di Mizio, G.; Murabito, P.; Carotenuto, M.; Messina, A.; Pisanelli, D.; et al. Functional Role of Dietary Intervention to Improve the Outcome of COVID-19: A Hypothesis of Work. Int. J. Mol. Sci. 2020, 21, 3104. [CrossRef] [PubMed]

3. Nishiura, H.; Jung, S.M.; Linton, N.M.; Kinoshita, R.; Yang, Y.; Hayashi, K.; Kobayashi, T.; Yuan, B.; Akhmetzhanov, A.R. Akhmetzhanov The Extent of Transmission of Novel Coronavirus in Wuhan, China, 2020. J. Clin. Med. 2020, 9, 330. [CrossRef]

4. Buja, L.M.; Barth, R.F.; Krueger, G.R.; Brodsky, S.V.; Hunter, R.L. The Importance of the Autopsy in Medicine: Perspectives of Pathology Colleagues. Acad. Pathol. 2019. [CrossRef] [PubMed]

5. Schwartz, D.A.; Herman, C.J. The importance of the autopsy in emerging and reemerging infectious diseases. Clin. Infect. Dis. 1996. [CrossRef] [PubMed]

6. Ng, D.L.; Al Hosani, F.; Keating, M.K.; Gerber, S.I.; Jones, T.L.; Metcalfe, M.G.; Tong, S.; Tao, Y.; Alami, N.N.; Haynes, L.M.; et al. Clinicopathologic, immunohistochemical, and ultrastructural findings of a fatal case of middle east respiratory syndrome coronavirus infection in the United Arab Emirates, April 2014. Am. J. Pathol. 2016. [CrossRef] [PubMed]

7. Liu, L.; Callinan, L.S.; Holman, R.C.; Blau, D.M. Determinants for autopsy after unexplained deaths possibly resulting from infectious causes, United States. Emerg. Infect. Dis. 2012. [CrossRef] 
8. Peng, Y.D.; Meng, K.; Guan, H.Q.; Leng, L.; Zhu, R.R.; Wang, B.Y.; He, M.A.; Cheng, L.X.; Huang, K.Z.Q. Clinical characteristics and outcomes of 112 cardiovascular disease patients infected by 2019-nCoV. Chin. J. Cardiovasc. Dis. 2020, 48. [CrossRef]

9. Tian, S.; Hu, W.; Niu, L.; Liu, H.; Xu, H.; Xiao, S.-Y. Pulmonary pathology of early phase 2019 novel coronavirus (COVID-19) pneumonia in two patients with lung cancer. J. Thorac. Oncol. 2020. [CrossRef]

10. Guan, W.; Ni, Z.; Hu, Y.; Liang, W.; Ou, C.; He, J.; Liu, L.; Shan, H.; Lei, C.; Hui, D.S.; et al. Clinical characteristics of 2019 novel coronavirus infection in China. medRxiv 2020. [CrossRef]

11. Xu, Z.; Shi, L.; Wang, Y.; Zhang, J.; Huang, L.; Zhang, C.; Liu, S.; Zhao, P.; Liu, H.; Zhu, L.; et al. Pathological findings of COVID-19 associated with acute respiratory distress syndrome. Lancet Respir. Med. 2020. [CrossRef]

12. Kui, L.; Fang, Y.-Y.; Deng, Y.; Liu, W.; Wang, M.-F.; Ma, J.-P.; Xiao, W.; Wang, Y.-N.; Zhong, M.-H.; Li, C.-H.; et al. Clinical characteristics of novel coronavirus cases in tertiary hospitals in Hubei Province. Chin. Med. J. (Engl.) 2020. [CrossRef]

13. Wang, W.; Tang, J.; Wei, F. Updated understanding of the outbreak of 2019 novel coronavirus (2019-nCoV) in Wuhan, China. J. Med. Virol. 2020. [CrossRef] [PubMed]

14. Yang, X.; Yu, Y.; Xu, J.; Shu, H.; Xia, J.; Liu, H.; Wu, Y.; Zhang, L.; Yu, Z.; Fang, M.; et al. Clinical course and outcomes of critically ill patients with SARS-CoV-2 pneumonia in Wuhan, China: a single-centered, retrospective, observational study. Lancet Respir. Med. 2020. [CrossRef]

15. Huang, C.; Wang, Y.; Li, X.; Ren, L.; Zhao, J.; Hu, Y.; Zhang, L.; Fan, G.; Xu, J.; Gu, X.; et al. Clinical features of patients infected with 2019 novel coronavirus in Wuhan, China. Lancet 2020. [CrossRef]

16. Wang, D.; Hu, B.; Hu, C.; Zhu, F.; Liu, X.; Zhang, J.; Wang, B.; Xiang, H.; Cheng, Z.; Xiong, Y.; et al. Clinical Characteristics of 138 Hospitalized Patients with 2019 Novel Coronavirus-Infected Pneumonia in Wuhan, China. JAMA - J. Am. Med. Assoc. 2020. [CrossRef]

17. Chen, N.; Zhou, M.; Dong, X.; Qu, J.; Gong, F.; Han, Y.; Qiu, Y.; Wang, J.; Liu, Y.; Wei, Y.; et al. Epidemiological and clinical characteristics of 99 cases of 2019 novel coronavirus pneumonia in Wuhan, China: a descriptive study. Lancet 2020. [CrossRef]

18. Zhang, Y.; Cao, W.; Xiao, M.; Li, Y.J.; Yang, Y.; Zhao, J.; Zhou, X.; Jiang, W.; Zhao, Y.Q.; Zhang, S.Y.; et al. Clinical and coagulation characteristics of 7 patients with critical COVID-2019 pneumonia and acro-ischemia. Zhonghua Xue Ye Xue Za Zhi 2020. [CrossRef]

19. Analysis on 54 mortality cases of Coronavirus disease 2019 in the Republic of Korea from January 19 to March 10, 2020. J. Korean Med. Sci. 2020. [CrossRef]

20. Yuan, M.; Yin, W.; Tao, Z.; Tan, W.; Hu, Y. Association of radiologic findings with mortality of patients infected with 2019 novel coronavirus in Wuhan, China. PLoS ONE 2020. [CrossRef]

21. Guo, T.; Fan, Y.; Chen, M.; Wu, X.; Zhang, L.; He, T.; Wang, H.; Wan, J.; Wang, X.; Lu, Z. Cardiovascular Implications of Fatal Outcomes of Patients with Coronavirus Disease 2019 (COVID-19). JAMA Cardiol. 2020. [CrossRef] [PubMed]

22. Li, Y.K.; Peng, S.; Li, L.Q.; Wang, Q.; Ping, W.; Zhang, N.; Fu, X.N. Clinical and Transmission Characteristics of Covid-19 - A Retrospective Study of 25 Cases from a Single Thoracic Surgery Department. Curr. Med. Sci. 2020. [CrossRef] [PubMed]

23. Chen, T.; Wu, D.; Chen, H.; Yan, W.; Yang, D.; Chen, G.; Ma, K.; Xu, D.; Yu, H.; Wang, H.; et al. Clinical characteristics of 113 deceased patients with coronavirus disease 2019: Retrospective study. BMJ 2020. [CrossRef] [PubMed]

24. Zhou, F.; Yu, T.; Du, R.; Fan, G.; Liu, Y.; Liu, Z.; Xiang, J.; Wang, Y.; Song, B.; Gu, X.; et al. Clinical course and risk factors for mortality of adult inpatients with COVID-19 in Wuhan, China: a retrospective cohort study. Lancet 2020. [CrossRef]

25. Deng, Y.; Liu, W.; Liu, K.; Fang, Y.Y.; Shang, J.; Zhou, L.; Wang, K.; Leng, F.; Wei, S.; Chen, L.; et al. Clinical characteristics of fatal and recovered cases of coronavirus disease 2019 (COVID-19) in Wuhan, China: A retrospective study. Chin. Med. J. (Engl) 2020. [CrossRef]

26. Rodriguez-Morales, A.J.; Cardona-Ospina, J.A.; Gutiérrez-Ocampo, E.; Villamizar-Peña, R.; Holguin-Rivera, Y.; Escalera-Antezana, J.P.; Alvarado-Arnez, L.E.; Bonilla-Aldana, D.K.; Franco-Paredes, C.; Henao-Martinez, A.F.; et al. Clinical, laboratory and imaging features of COVID-19: A systematic review and meta-analysis. Travel Med. Infect. Dis. 2020. [CrossRef] 
27. Guan, W.; Liang, W.; Zhao, Y.; Liang, H.; Chen, Z.; Li, Y.; Liu, X.; Chen, R.; Tang, C.; Wang, T.; et al. Comorbidity and its impact on 1590 patients with Covid-19 in China: A Nationwide Analysis. Eur. Respir. J. 2020. [CrossRef]

28. Bhatraju, P.K.; Ghassemieh, B.J.; Nichols, M.; Kim, R.; Jerome, K.R.; Nalla, A.K.; Greninger, A.L.; Pipavath, S.; Wurfel, M.M.; Evans, L.; et al. Covid-19 in Critically Ill Patients in the Seattle Region-Case Series. N. Engl. J. Med. 2020. [CrossRef]

29. Zhang, L.; Zhu, F.; Xie, L.; Wang, C.; Wang, J.; Chen, R.; Jia, P.; Guan, H.Q.; Peng, L.; Chen, Y.; et al. Clinical characteristics of COVID-19-infected cancer patients: A retrospective case study in three hospitals within Wuhan, China. Ann. Oncol. 2020. [CrossRef]

30. Lescure, F.X.; Bouadma, L.; Nguyen, D.; Parisey, M.; Wicky, P.H.; Behillil, S.; Gaymard, A.; Bouscambert-Duchamp, M.; Donati, F.; Le Hingrat, Q.; et al. Clinical and virological data of the first cases of COVID-19 in Europe: a case series. Lancet. Infect. Dis. 2020. [CrossRef]

31. Wu, C.; Chen, X.; Cai, Y.; Xia, J.; Zhou, X.; Xu, S.; Huang, H.; Zhang, L.; Zhou, X.; Du, C.; et al. Risk Factors Associated With Acute Respiratory Distress Syndrome and Death in Patients With Coronavirus Disease 2019 Pneumonia in Wuhan, China. JAMA Intern. Med. 2020. [CrossRef] [PubMed]

32. Grasselli, G.; Zangrillo, A.; Zanella, A.; Antonelli, M.; Cabrini, L.; Castelli, A.; Cereda, D.; Coluccello, A.; Foti, G.; Fumagalli, R.; et al. Baseline Characteristics and Outcomes of 1591 Patients Infected with SARS-CoV-2 Admitted to ICUs of the Lombardy Region, Italy. JAMA - J. Am. Med. Assoc. 2020. [CrossRef] [PubMed]

33. Mi, B.; Chen, L.; Xiong, Y.; Xue, H.; Zhou, W.; Liu, G. Characteristics and Early Prognosis of COVID-19 Infection in Fracture Patients. J. Bone Jt. Surg. 2020. [CrossRef] [PubMed]

34. Chen, T.; Dai, Z.; Mo, P.; Li, X.; Ma, Z.; Song, S.; Chen, X.; Luo, M.; Liang, K.; Gao, S.; et al. Clinical characteristics and outcomes of older patients with coronavirus disease 2019 (COVID-19) in Wuhan, China (2019): A single-centered, retrospective study. Journals Gerontol. Ser. A 2020. [CrossRef] [PubMed]

35. Li, X.; Wang, L.; Yan, S.; Yang, F.; Xiang, L.; Zhu, J.; Shen, B.; Gong, Z. Clinical characteristics of 25 death cases with COVID-19: a retrospective review of medical records in a single medical center, Wuhan, China. Int. J. Infect. Dis. 2020. [CrossRef] [PubMed]

36. Cao, J.; Tu, W.J.; Cheng, W.; Yu, L.; Liu, Y.K.; Hu, X.; Liu, Q. Clinical Features and Short-term Outcomes of 102 Patients with Corona Virus Disease 2019 in Wuhan, China. Clin. Infect. Dis. 2020. [CrossRef]

37. Wang, L.; He, W.; Yu, X.; Hu, D.; Bao, M.; Liu, H.; Zhou, J.; Jiang, H. Coronavirus disease 2019 in elderly patients: Characteristics and prognostic factors based on 4-week follow-up. J. Infect. 2020. [CrossRef]

38. Barton, L.M.; Duval, E.J.; Stroberg, E.; Ghosh, S.; Mukhopadhyay, S. COVID-19 Autopsies, Oklahoma, USA. Am. J. Clin. Pathol. 2020. [CrossRef]

39. Huang, J.; Lin, H.; Wu, Y.; Fang, Y.; Kumar, R.; Chen, G.; Lin, S. COVID-19 in posttransplant patients-Report of 2 cases. Am. J. Transplant. 2020. [CrossRef]

40. Ling, L.; So, C.; Shum, H.P.; Chan, P.K.S.; Lai, C.K.C.; Kandamby, D.H.; Ho, E.; So, D.; Yan, W.W.; Lui, G.; et al. Critically ill patients with COVID-19 in Hong Kong: A multicentre retrospective observational cohort study. Crit. Care Resusc. 2020, 32248675, Online ahead of print.

41. Cheng, Y.; Luo, R.; Wang, K.; Zhang, M.; Wang, Z.; Dong, L.; Li, J.; Yao, Y.; Ge, S.; Xu, G. Kidney disease is associated with in-hospital death of patients with COVID-19. Kidney Int. 2020. [CrossRef] [PubMed]

42. Du, R.H.; Liang, L.R.; Yang, C.Q.; Wang, W.; Cao, T.Z.; Li, M.; Guo, G.Y.; Du, J.; Zheng, C.L.; Zhu, Q.; et al. Predictors of Mortality for Patients with COVID-19 Pneumonia Caused by SARS-CoV-2: A Prospective Cohort Study. Eur. Respir. J. 2020. [CrossRef] [PubMed]

43. Barrasa, H.; Rello, J.; Tejada, S.; Martín, A.; Balziskueta, G.; Vinuesa, C.; Fernández-Miret, B.; Villagra, A.; Vallejo, A.; Sebastián, A.S.; et al. SARS-Cov-2 in Spanish Intensive Care: Early Experience with 15-day Survival In Vitoria. Anaesth. Crit. Care Pain Med. 2020. [CrossRef]

44. Lovell, N.; Maddocks, M.; Etkind, S.N.; Taylor, K.; Carey, I.; Vora, V.; Marsh, L.; Higginson, I.J.; Prentice, W.; Edmonds, P.; et al. Characteristics, symptom management and outcomes of 101 patients with COVID-19 referred for hospital palliative care. J. Pain Symptom Manage. 2020. [CrossRef] [PubMed]

45. Wang, X.; Liu, W.; Zhao, J.; Lu, Y.; Wang, X.; Yu, C.; Hu, S.; Shen, N.; Liu, W.; Sun, Z.; et al. Clinical characteristics of 80 hospitalized frontline medical workers infected with COVID-19 in Wuhan, China. J. Hosp. Infect. 2020. [CrossRef]

46. Zhang, G.; Hu, C.; Luo, L.; Fang, F.; Chen, Y.; Li, J.; Peng, Z.; Pan, H. Clinical features and short-term outcomes of 221 patients with COVID-19 in Wuhan, China. J. Clin. Virol. 2020. [CrossRef] 
47. Magro, C.; Mulvey, J.J.; Berlin, D.; Nuovo, G.; Salvatore, S.; Harp, J.; Baxter-Stoltzfus, A.; Laurence, J. Complement associated microvascular injury and thrombosis in the pathogenesis of severe COVID-19 infection: A report of five cases. Transl. Res. 2020. [CrossRef]

48. Pereira, M.R.; Mohan, S.; Cohen, D.J.; Husain, S.A.; Dube, G.K.; Ratner, L.E.; Arcasoy, S.; Aversa, M.M.; Benvenuto, L.J.; Dadhani, D.; et al. COVID-19 in Solid Organ Transplant Recipients: Initial Report from the US Epicenter. Am. J. Transplant. 2020. [CrossRef]

49. Li, J.; Wang, X.; Chen, J.; Zuo, X.; Zhang, H.; Deng, A. COVID-19 infection may cause ketosis and ketoacidosis. Diabetes Obes. Metab. 2020. [CrossRef]

50. Yang, F.; Shi, S.; Zhu, J.; Shi, J.; Dai, K.; Chen, X. Analysis of 92 deceased patients with COVID-19. J. Med. Virol. 2020. [CrossRef]

51. Cai, Y.; Hao, Z.; Gao, Y.; Ping, W.; Wang, Q.; Peng, S.; Zhao, B.; Sun, W.; Zhu, M.; Li, K.; et al. Coronavirus Disease 2019 in the Perioperative Period of Lung Resection: A Brief Report From a Single Thoracic Surgery Department in Wuhan, People's Republic of China. J. Thorac. Oncol. 2020. [CrossRef] [PubMed]

52. Karami, P.; Naghavi, M.; Feyzi, A.; Aghamohammadi, M.; Novin, M.S.; Mobaien, A.; Qorbanisani, M.; Karami, A.; Norooznezhad, A.H. Mortality of a pregnant patient diagnosed with COVID-19: A case report with clinical, radiological, and histopathological findings. Travel Med. Infect. Dis. 2020, 101665. [CrossRef] [PubMed]

53. Tian, S.; Xiong, Y.; Liu, H.; Niu, L.; Guo, J.; Liao, M.; Xiao, S.-Y. Pathological study of the 2019 novel coronavirus disease (COVID-19) through postmortem core biopsies. Mod. Pathol. 2020. [CrossRef]

54. Fabre, O.; Rebet, O.; Carjaliu, I.; Radutoiu, M.; Gautier, L.; Hysi, I. Severe Acute Proximal Pulmonary Embolism and COVID-19: A Word of Caution. Ann. Thorac. Surg. 2020. [CrossRef] [PubMed]

55. Su, H.; Yang, M.; Wan, C.; Yi, L.X.; Tang, F.; Zhu, H.Y.; Yi, F.; Yang, H.C.; Fogo, A.B.; Nie, X.; et al. Renal histopathological analysis of 26 postmortem findings of patients with COVID-19 in China. Kidney Int. 2020. [CrossRef] [PubMed]

56. Edrada, E.M.; Lopez, E.B.; Villarama, J.B.; Salva Villarama, E.P.; Dagoc, B.F.; Smith, C.; Sayo, A.R.; Verona, J.A.; Trifalgar-Arches, J.; Lazaro, J.; et al. First COVID-19 infections in the Philippines: A case report. Trop. Med. Health 2020. [CrossRef] [PubMed]

57. Hang, L.; Yan, X.; Fan, Q.; Liu, H.; Liu, X.; Liu, Z.; Zhang, Z. D-dimer levels on admission to predict in-hospital mortality in patients with Covid-19. J. Thromb. Haemost. 2020. [CrossRef]

58. Zheng, Y.-Y.; Ma, Y.-T.; Zhang, J.-Y.; Xie, X. COVID-19 and the cardiovascular system. Nat. Rev. Cardiol. 2020. [CrossRef]

59. Rotzinger, D.C.; Beigelman-Aubry, C.; von Garnier, C.; Qanadli, S.D. Pulmonary embolism in patients with COVID-19: Time to change the paradigm of computed tomography. Thromb. Res. 2020, 190, 58-59. [CrossRef]

60. Klok, F.A.; Kruip, M.J.H.A.; van der Meer, N.J.M.; Arbous, M.S.; Gommers, D.A.M.P.J.; Kant, K.M.; Kaptein, F.H.J.; van Paassen, J.; Stals, M.A.M.; Huisman, M.V.; et al. Incidence of thrombotic complications in critically ill ICU patients with COVID-19. Thromb. Res. 2020. [CrossRef]

61. Mehta, P.; McAuley, D.F.; Brown, M.; Sanchez, E.; Tattersall, R.S.; Manson, J.J. COVID-19: Consider cytokine storm syndromes and immunosuppression. Lancet 2020. [CrossRef]

62. World Health Organization. Infection Prevention and Control for the safe management of a dead body in the context of COVID-19. Interim guidance: 24 March 2020. Available online: https:/apps.who.int/iris/bitstream/ handle/10665/331538/WHO-COVID-19-1PC_DBMgmt-2020.1-eng.pdf (accessed on 27 March 2020).

63. Italian Ministry of Health. Emergency Indications Related to the COVID-19 Epidemic Concerning the Funeral Sector, Cemetery, and Cremation. Circular of General Direction of Health Prevention. 2020. Available online: http://www.trovanorme.salute.gov.it/norme/renderNormsanPdf?anno=2020\&codLeg=73965\&parte= 1\%20\&serie=null (accessed on 3 May 2020).

64. Hanley, B.; Lucas, S.B.; Youd, E.; Swift, B.; Osborn, M. Autopsy in suspected COVID-19 cases. J. Clin. Pathol. 2020. [CrossRef]

65. Pomara, C.; Volti, G.L.; Cappello, F. COVID-19 Deaths: Are We Sure It Is Pneumonia? Please, Autopsy, Autopsy, Autopsy! J. Clin. Med. 2020, 9, 1259. [CrossRef] [PubMed]

66. De Cock, K.M.; Zielinski-Gutiérrez, E.; Lucas, S.B. Learning from the Dead. N. Engl. J. Med. 2019. [CrossRef]

67. Vetter, P.; Fischer, W.A.; Schibler, M.; Jacobs, M.; Bausch, D.G.; Kaiser, L. Ebola Virus Shedding and Transmission: Review of Current Evidence. J. Infect. Dis. 2016. [CrossRef] [PubMed] 
68. Castillo, P.; Martínez, M.J.; Ussene, E.; Jordao, D.; Lovane, L.; Ismail, M.R.; Carrilho, C.; Lorenzoni, C.; Fernandes, F.; Bene, R.; et al. Validity of a Minimally Invasive Autopsy for Cause of Death Determination in Adults in Mozambique: An Observational Study. PLoS Med. 2016. [CrossRef] [PubMed]

69. Kreuels, B.; Wichmann, D.; Emmerich, P.; Schmidt-Chanasit, J.; De Heer, G.; Kluge, S.; Sow, A.; Renné, T.; Günther, S.; Lohse, A.W.; et al. A case of severe Ebola virus infection complicated by gram-negative septicemia. N. Engl. J. Med. 2014. [CrossRef] [PubMed]

70. Kreuels, B.; Addo, M.M.; Schmiedel, S. Severe Ebola virus infection complicated by gram-negative septicemia. N. Engl. J. Med. 2015. [CrossRef]

71. Petrosillo, N.; Nicastri, E.; Lanini, S.; Capobianchi, M.R.; Di Caro, A.; Antonini, M.; Puro, V.; Lauria, F.N.; Shindo, N.; Magrini, N.; et al. Ebola virus disease complicated with viral interstitial pneumonia: A case report. BMC Infect. Dis. 2015. [CrossRef]

72. Varkey, J.B.; Shantha, J.G.; Crozier, I.; Kraft, C.S.; Lyon, G.M.; Mehta, A.K.; Kumar, G.; Smith, J.R.; Kainulainen, M.H.; Whitmer, S.; et al. Persistence of ebola virus in ocular fluid during convalescence. N. Engl. J. Med. 2015. [CrossRef]

73. Brief Report: Persistence of Ebola Virus in Ocular Fluid during Convalescence. N. Engl. J. Med. 2015. [CrossRef]

74. Francis, T.I.; Moore, D.L.; Edington, G.M.; Smith, J.A. A clinicopathological study of human yellow fever. Bull. World Health Organ. 1972, 46, 659. [PubMed]

75. Duarte-Neto, A.N.; Monteiro, R.A.; Johnsson, J.; Cunha, M.; Pour, S.Z.; Saraiva, A.C.; Ho, Y.L.; da Silva, L.F.F.; Mauad, T.; Zanotto, P.M.; et al. Ultrasound-guided minimally invasive autopsy as a tool for rapid post-mortem diagnosis in the 2018 Sao Paulo yellow fever epidemic: Correlation with conventional autopsy. PLoS Negl. Trop. Dis. 2019. [CrossRef]

76. Burton, J.L.; Underwood, J. Clinical, educational, and epidemiological value of autopsy. Lancet 2007. [CrossRef]

77. Franks, T.J.; Chong, P.Y.; Chui, P.; Galvin, J.R.; Lourens, R.M.; Reid, A.H.; Selbs, E.; Mcevoy, C.P.L.; Hayden, C.D.L.; Fukuoka, J.; et al. Lung pathology of severe acute respiratory syndrome (SARS): A study of 8 autopsy cases from Singapore. Hum. Pathol. 2003. [CrossRef]

78. Roosen, J.; Frans, E.; Wilmer, A.; Knockaert, D.C.; Bobbaers, H. Comparison of premortem clinical diagnoses in critically ill patients and subsequent autopsy findings. Mayo Clin. Proc. 2000. [CrossRef]

79. Kotovicz, F.; Mauad, T.; Saldiva, P.H.N. Clinico-pathological discrepancies in a general university hospital in São Paulo, Brazil. Clinics 2008. [CrossRef]

80. Combes, A.; Mokhtari, M.; Couvelard, A.; Trouillet, J.L.; Baudot, J.; Hénin, D.; Gibert, C.; Chastre, J. Clinical and Autopsy Diagnoses in the Intensive Care Unit: A Prospective Study. Arch. Intern. Med. 2004. [CrossRef]

81. Edwards, J.E. The Autopsy: Do We Still Need It? Chest 1970, 57, 113-114. [CrossRef] 